\title{
A third anti-SARS-CoV-2 mRNA dose does not overcome the pejorative impact of anti-CD20 therapy and/or low immunoglobulin levels in patients with lymphoma or chronic lymphocytic leukemia
}

Patients with non-Hodgkin lymphoma (NHL) or chronic lymphocytic leukemia (CLL) share immune deficiencies due to the biological features of these diseases and their treatment. They are at risk of developing severe and/or prolonged forms of coronavirus disease 2019 (COVID-19)., ${ }^{1,2}$ Moreover, their seroconversion rate after infection by the etiological agent, severe acute respiratory syndrome coronavirus-2 (SARS-CoV-2) or after vaccination is low, in particular, after recently receiving anti-CD20 monoclonal antibodies. ${ }^{3,4}$ Addition of a third vaccine dose is, therefore, recommended for non-responding patients or those with a low serological response. Here, we show that the addition of a third dose does not modify the proportion of seropositivity after vaccination in a population of patients with $\mathrm{NHL} / \mathrm{CLL}$ when treated with anti-CD20 therapies.

The antibody response to mRNA SARS-CoV-2 vaccine in $\mathrm{NHL} / \mathrm{CLL}$ patients has been shown to be negatively affected by their treatments, in particular, when treatment is ongoing during the vaccination. In a recent cohort of nearly $400 \mathrm{CLL}$ patients, the seropositivity rate was $61 \%$ in treatment-naïve patients versus $23 \%$ in patients treated with Bruton tyrosine kinase (BTK) inhibitors, 24\% in those treated with Bcl2 inhibitors, and, remarkably, only $5 \%$ in those who received anti-CD20 monoclonal antibodies in the year before vaccination. ${ }^{4}$ The results were similar among patients with NHL., ${ }^{5,6}$ In a cohort of 162 lymphoma patients, the proportion of seropositivity was $51 \%$ overall and decreased to $12 \%$ within the first 12 months following treatment with anti-CD20 antibodies. ${ }^{5}$ Boosting solid organ transplanted patients, who are at risk of severe COVID-19 after two doses of vaccine, ${ }^{7}$ with a third dose of mRNA vaccine was shown to significantly improve their immune response (seropositivity of 55\% after third dose versus $18 \%$ in a placebo group). ${ }^{8.9} \mathrm{Al}-$ though, the addition of a third dose of vaccine for immunocompromised patients has been recommended in France since April 2021, there has been no evaluation of this strategy in patients with hematologic malignancies. We therefore conducted a single-center study in our institution to investigate determinants of the antibody response to anti-SARS-CoV-2 Pfizer-BioNTech ${ }^{\circledR}$ BNT162b2 or Moderna ${ }^{\circledR}$ mRNA-1273 vaccines among adults with a current or past diagnosis of lymphoma/CLL. The addition of a third dose was at the discretion of each physician. The two-dose vaccination courses were Pfizer $(n=58)$ and
Moderna $(n=6)$ and the three-dose vaccination courses were Pfizer $(n=35)$ and Moderna/Moderna/Pfizer $(n=1)$. The median interval between the first and second vaccine doses was 28 days while that between the second and third vaccine doses was 61 days. Serology was performed at least 2 weeks after the last vaccination.

Data were extracted from the patients' medical charts and included their demographics, lymphoma/CLL history and treatment, vaccination dates and biological variables (immunoglobulin [Ig] G level, total lymphocytes, B-cell, and T-cell counts [determined by a Beckman Coulter ${ }^{\circledR}$ Aquios Tetra system], and SARS-CoV-2 anti-spike serology (determined by ECLIA Elecsys anti-SARS-CoV2 S, Roch ${ }^{\circledR}{ }^{\circledR}$ ). Negative serological tests were defined as antibody titers $<0.8 \mathrm{U} / \mathrm{mL}$. The patients' baseline characteristics are presented using medians and interquartile ranges (IQR) or ranges for quantitative variables and frequencies and percentages for categorical variables. The association between patients' serological status and other variables was assessed using a Wilcoxon or Fisher test, as appropriate, and univariate logistic regression. Multivariate logistic regression was performed for variables for which the univariate two-sided significance was $<5 \%$ and for which there was a minimum of two patients per category for categorical variables (for reasons of algorithmic convergence). Statistical analysis was performed using $R$ and the environment for statistical computing version 4.03 ( $R$ Core Team, 2020). The study was conducted in accordance with the Declaration of Helsinki, and approved by the ethics committee of our institution.

In total, 100 patients with B-cell NHL $(n=51)$, T-cell NHL $(n=4), C L L(n=33)$ or Hodgkin lymphoma $(H L)(n=12)$ were analyzed (Table 1). The median age of the patients was 66.6 (range, 19.6-92.2) years and the female/male ratio was 0.43 . The median interval between serology and the last vaccine injection (second or third) was 47 days. Fiftyone patients $(51 \%)$ showed negative serology, with the proportion of seronegativity being $55 \%, 50 \%, 67 \%$ and $39 \%$ in patients with B-cell NHL, T-cell NHL, HL and CLL, respectively $(P=0.35)$. Ten patients had a history of COVID-19, and of these, three needed hospitalization. One of these patients developed COVID-19 after failing to seroconvert following his second vaccine injection. Patients with negative serology had significantly lower lymphocyte 
Table 1. Characteristics of patents with lymphoma/chronic lymphocytic leukemia according to their serological response to anti-SARS-CoV-2 vaccination.

\begin{tabular}{|c|c|c|c|c|c|c|}
\hline & \multirow{3}{*}{$\begin{array}{l}\text { All patients, } \\
\text { n. } 100\end{array}$} & \multirow{3}{*}{$\begin{array}{c}\text { Positive } \\
\text { serology, } \\
\text { n (\%) } \\
49(49.0)\end{array}$} & \multirow{3}{*}{$\begin{array}{c}\text { Negative } \\
\text { serology, } \\
\text { n (\%) } \\
51(51.0)\end{array}$} & \multicolumn{3}{|c|}{$\begin{array}{l}\text { OR }(95 \% \mathrm{CI}) \\
\text { P value }\end{array}$} \\
\hline & & & & \multirow[t]{2}{*}{ Univariable } & \multicolumn{2}{|c|}{ Multivariable } \\
\hline & & & & & model 1 & model 2 \\
\hline \multicolumn{7}{|l|}{$\begin{array}{l}\text { Demographic } \\
\text { characteristics }\end{array}$} \\
\hline $\begin{array}{l}\text { Age in years, median } \\
\text { (IQR) }\end{array}$ & $\begin{array}{c}66.9 \\
(54.9-76.9)\end{array}$ & $\begin{array}{c}66.6 \\
(54.9-76.9)\end{array}$ & $\begin{array}{c}67.0 \\
(56.3-77.0)\end{array}$ & $\begin{array}{c}1.00 \\
(0.97-1.02) \\
P=0.76\end{array}$ & & \\
\hline \multicolumn{7}{|l|}{ Gender } \\
\hline Female & 30 & $15(50.0)$ & $15(50.0)$ & 1.06 & & \\
\hline Male & 70 & $34(48.6)$ & $36(51.4)$ & $P=0.90$ & & \\
\hline \multicolumn{7}{|l|}{ Previous COVID-19 } \\
\hline No & 90 & $40(45.1)$ & $50(54.9)$ & 0.09 & $\mathrm{NA}$ & $\mathrm{NA}$ \\
\hline Yes & 10 & $9(90.0)$ & $1(10.0)$ & $P=0.02$ & I & 4 \\
\hline \multicolumn{7}{|l|}{$\begin{array}{l}\text { N. of vaccine } \\
\text { injections }\end{array}$} \\
\hline 2 & & & & $\begin{array}{c}1.59 \\
(0.70-3.67)\end{array}$ & $\begin{array}{c}1.41 \\
(0.47-4.32)\end{array}$ & $\begin{array}{c}1.32 \\
(0.46-3.77)\end{array}$ \\
\hline 3 & 36 & $15(41.7)$ & $21(58.3)$ & $P=0.27$ & $P=0.54$ & $P=0.61$ \\
\hline $\begin{array}{l}\text { N. of days between } \\
\text { first vaccination and } \\
\text { serology, median } \\
(\text { IQR) }\end{array}$ & $\begin{array}{c}47.5 \\
(33.0-78.25)\end{array}$ & $\begin{array}{c}53.0 \\
(37.0-84.0)\end{array}$ & $\begin{array}{c}45.0 \\
(28.5-72.0)\end{array}$ & $\begin{array}{c}1.00 \\
(0.99-1.00) \\
P=0.30\end{array}$ & & \\
\hline \multicolumn{7}{|l|}{$\begin{array}{l}\text { Lymphoma } \\
\text { characteristics }\end{array}$} \\
\hline B-NHL & 51 & $23(45.1)$ & $28(54.9)$ & reference & & \\
\hline T-NHL & 4 & $2(50.0)$ & $2(50.0)$ & $\begin{array}{c}0.82 \\
(0.09-7.28) \\
P=0.85\end{array}$ & & \\
\hline$H L$ & 12 & 4 (33.3) & $8(66.7)$ & $\begin{array}{c}1.64 \\
(0.46-6.80) \\
P=0.46\end{array}$ & & \\
\hline CLL & 33 & $20(60.6)$ & $13(39.4)$ & $\begin{array}{c}0.53 \\
(0.22-1.29) \\
P=0.17\end{array}$ & & \\
\hline
\end{tabular}

\section{Biological}

characteristics

Neutrophils*

Median (IQR)

$\geq 1.5 \times 10 \% / \mathrm{L}$

$<1.5 \times 109 / \mathrm{L}$

Lymphocytes**

Median, IQR

$\geq 1.5 \times 10 \% / L$

$<1.5 \times 10^{9} / \mathrm{L}$

T cells ${ }^{\star \star \star}$

Median, IQR

3.7
$(2.2-4.9)$
81
10

1.0

(0.6-2.0)

35

55

0.6

(0.3-1.2)
3.8

(2.83-5.4)

41 (50.6)

$2(20.0)$
3.4

(1.9-4.7)

40 (49.4)

$8(80.0)$

0.8

(0.5-1.4)

12 (34.3)

35 (63.6)

20 (36.4)

0.7

(0.4-1.4)
0.5

(0.3-1.1) reference

4.10

(0.96-8.29)

$P=0.09$

reference

3.35

(1.40-8.37)

$P<0.01$ 


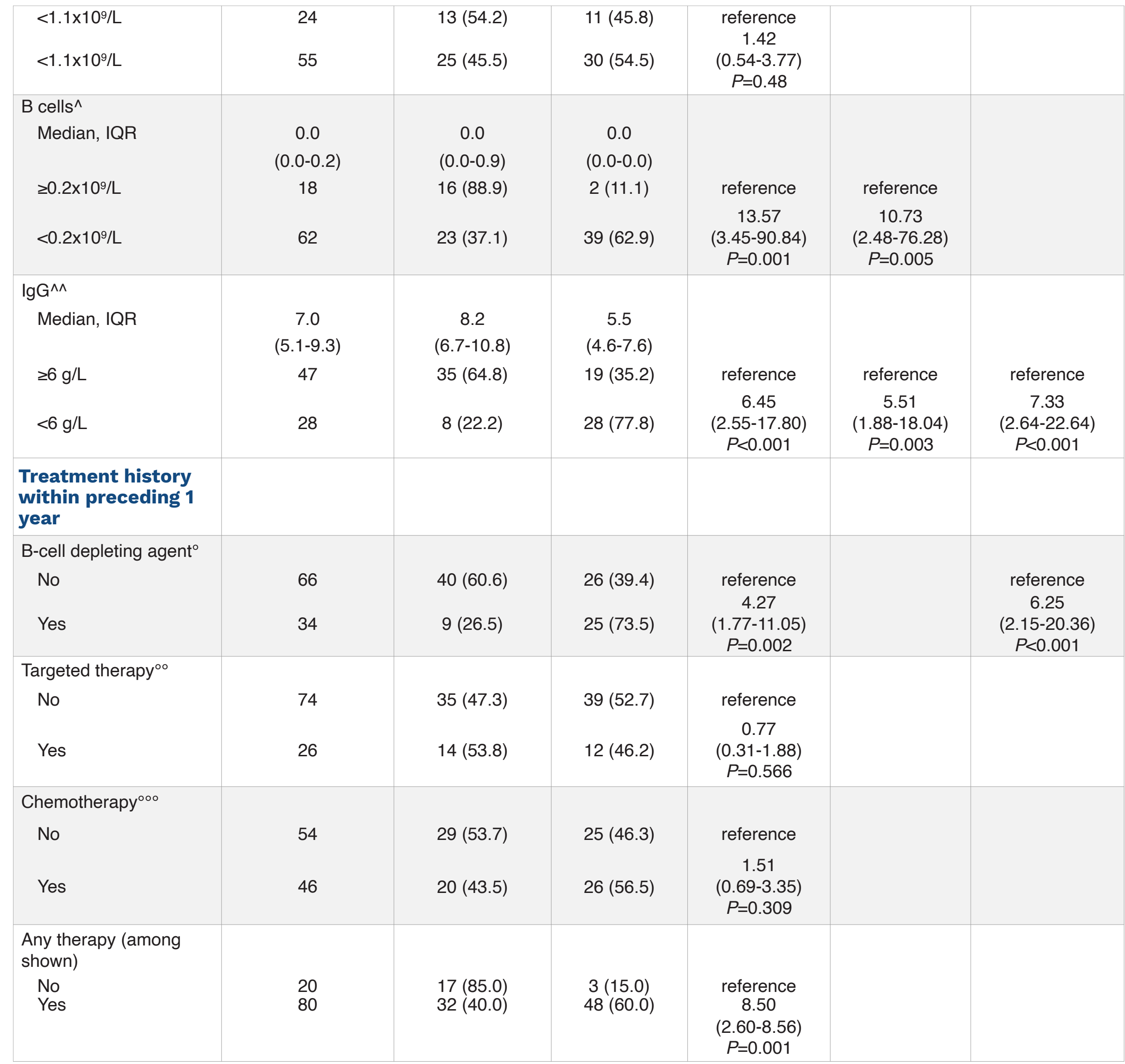

Missing values: $* 9, * * 10, * * * 21, \wedge 20, \wedge \wedge 26 .{ }^{\circ}$ Rituximab $(n=30)$, obinutuzumab $(n=4)$; ooibrutinib and/or venetoclax ( $\left.n=20\right)$, masitinib ( $\left.n=1\right)$, pembrolizumab $(n=3)$, lenalidomide $(n=2)$; ${ }^{\circ{ }^{\circ} R-C H O P}$ and R-CHOP-like $(n=39)$, R-DHAX $(n=7)$; R-bendamustine $(n=7)$, ABVD $(n=6)$, BEACOPP $(n=4)$, rituximab/fludarabin/cyclophosphamide $(n=4)$, the total exceeds the number of patients since some patients received more than one line of chemotherapy. OR: odds ratio; 95\% Cl: 95\% confidence interval; IQR: interquartile range; COVID-19: coronavirus disease 2019; NHL: non-Hodgkin lymphoma; HL. Hodgkin lymphoma; CLL: chronic lymphocytic leukemia; NA: not available because of the absence of algorithmic convergence.

counts (median $0.8 \times 10^{\%} / \mathrm{L}$ versus $1.4 \times 10^{9} / \mathrm{L} ; P=0.006$ ), Bcell counts (median $0.00 \times 10^{9} / \mathrm{L}$ versus $0.04 \times 10^{9} / \mathrm{L} ; P<0.001$ ) and IgG levels (median $5.5 \times 10^{9} / \mathrm{L}$ versus $8.2 \times 10^{\circ} / \mathrm{L}$; $P<0.001)$ than seropositive patients. They were also less likely to have a history of COVID-19: odds ratio $(O R)=0.09$, 95\% confidence interval (95\% Cl): 0.00-0.50; $P=0.02$. Patients who had received any treatment within the year before their first vaccine injection (anti-CD20 [n=34], chemotherapy $[n=46]$, or targeted therapy $[n=26]$ ) had a higher risk of seronegativity than other patients $(n=20)$ (OR=8.50 [95\% Cl: 2.60-38.56]; $P=0.001)$. Among treatments, anti-CD20 was strongly associated with negative serology (OR=4.27, [95\% Cl: 1.77-11.05]; $P=0.002)$. Time between first vaccine injection and anti-CD20 administration was significantly associated with patients' serological status $\left(P<10^{-3}\right)$. Most patients who had their last antiCD20 administration within 1 year prior to first vaccine injection did not seroconvert (25 out of $34,74 \%$ ). Out of 


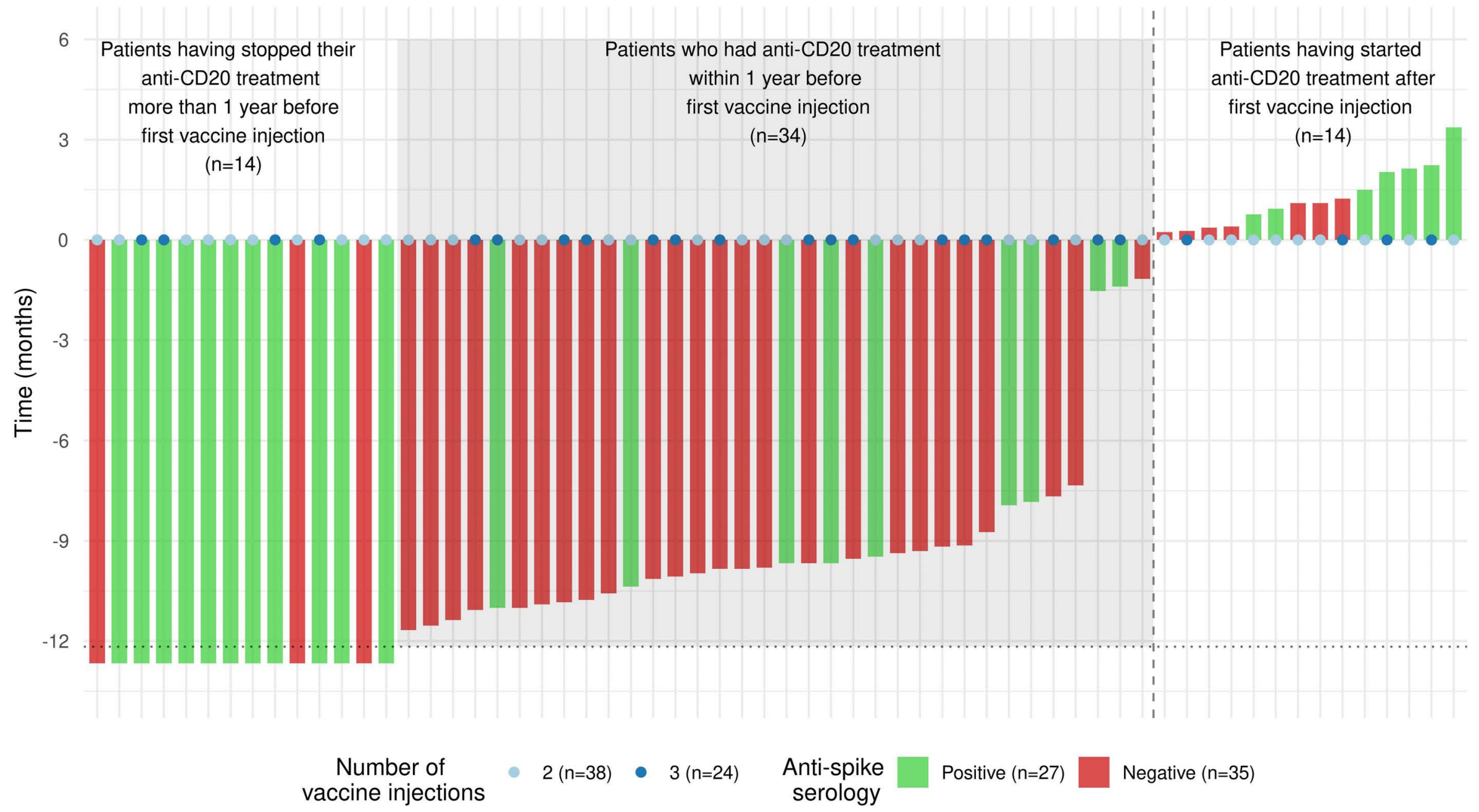

Figure 1. Time interval between the first vaccine injection and the last (if prior to vaccination) or first (if after vaccination) antiCD20 administration. Time is represented by vertical bars and the color code represents patients' serological status. Patients who had their last anti-CD20 administration before their first vaccine injection are represented at the left hand side of the vertical dotted line. Patients who had their last anti-CD20 administration after their first vaccine injection are represented at the right hand side of the vertical dotted line. The color code of the vertical bars represents patients' serological status. The total number of vaccine doses received prior to serology is represented by colored points (2 doses or 3 doses).

these 25 negative patients, $12(48 \%)$ had three vaccine injections (Figure 1). Among the nine patients who seroconverted, four had received three injections (44\%). Among the 14 patients who had their first anti-CD20 administration after their first vaccine injection, seven did not seroconvert. Of note these seven patients had their first anti-CD20 administration within 45 days after their first vaccine injection.

Overall, there was no association between the number of vaccine injections and seroconversion: $58.3 \%$ of patients who had three doses remained seronegative versus $46.9 \%$ of patients who had two doses (OR=1.59 [95\% Cl: $0.70-3.67] ; P=0.27)$. The titers of anti-spike serology according to anti-CD20 status and the number of vaccine administrations are presented in Figure 2. Regardless of the number of vaccine administrations, patients who had their last anti-CD20 administration within the year before the first vaccine injection had low anti-spike titers (median titer: $0.4 \mathrm{U} / \mathrm{mL}$ ), patients who had their first antiCD20 administration after their first vaccine injection had intermediate titers of anti-spike (median titer: $1.0 \mathrm{u} / \mathrm{mL}$ ), while other patients had higher levels of anti-spike (median titer: $47.6 \mathrm{U} / \mathrm{mL}$ ). As anti-CD20 administration and B-cell counts correlated strongly, we performed multivariable analyses using two models, model 1 based on B-cell counts, and model 2, based on anti-CD20. Low IgG levels (OR=7.33, [95\% Cl: 2.64-22.64]; $P<0.001)$ and recent anti-CD20 treatment (OR=6.25, [95\% Cl: 2.15-20.36]; $P<0.001)$ were both associated with seronegativity. The results were similar with low $\mathrm{B}$-cell counts (Table 1 ).

Overall, recent anti-CD20 therapy (low B-cell counts) and low IgG levels are the main independent factors associated with a poor serological response after anti-SARSCoV-2 mRNA vaccination in CLL/lymphoma patients. Administration of a third vaccine dose did not overcome the poor serological response observed in patients who had anti-CD20 treatment within 1 year prior to their first vaccine injection or low IgG levels. However, due to its single-center character, the limited number of patients and the heterogeneity of the lymphoid malignancies described, results of our study should be confirmed by other studies. Moreover, clinical studies examining the impact of the lack of serological conversion on the incidence and severity of COVID-19 are needed. Indeed, several studies have reported the presence of T-cell responses after vaccination in patients treated with rituximab for autoimmune diseases, ${ }^{10-12}$ despite an altered humoral response and low seroconversion levels. Never- 

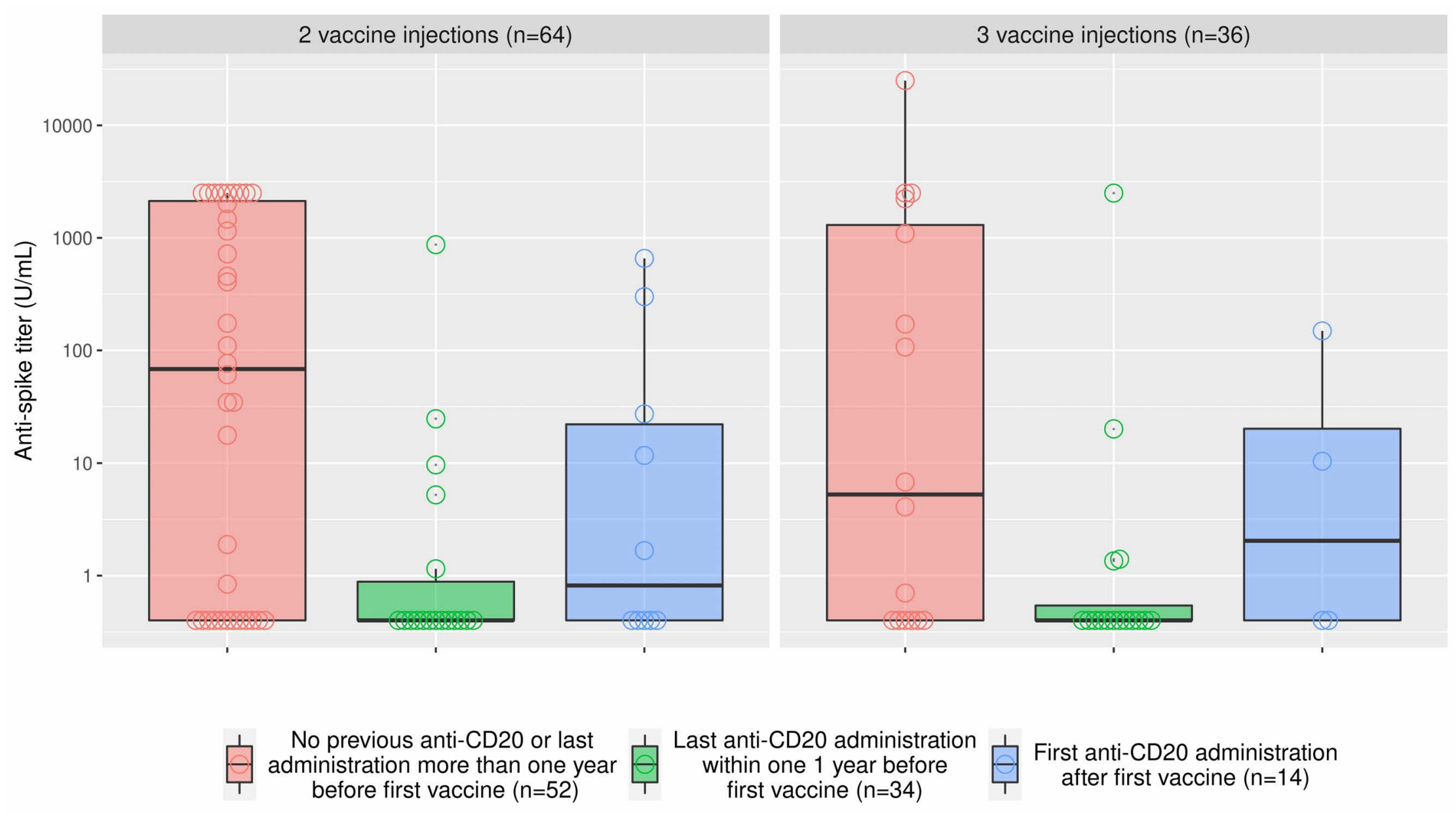

Figure 2. Anti-spike titers with respect to anti-CD20 treatment and the number of vaccine injections. The natural logarithm of the anti-spike titer is represented with respect to anti-CD20 treatment status. Red: patients without a history of anti-CD20 treatment or patients who had their last administration more than 1 year prior to the first vaccine injection; green: patients who had their last anti-CD20 administration within 1 year prior to the first vaccine injection; and (blue) patients who had their first vaccine injection prior to their first anti-CD20 administration.

theless, the clinical benefit of the cellular immune response in COVID-19 is yet to be elucidated. The minimum time between the last dose of anti-CD20 and vaccination $^{3,5}$ to observe patients' serological response remains controversial: in line with our results previously published studies have suggested a minimum of 12 months whereas others have reported a time interval of 3 months..$^{13}$ In the meantime, strategies other than increasing the number of vaccine injections are needed to protect patients with B-cell depletion against COVID-19. First, SARS-CoV-2 vaccination should be proposed before the onset of lymphoma/CLL therapy in all non-critical clinical situations. Strategies of maintenance treatment in follicular and mantle-cell lymphoma with anti-CD20 therapy should be discussed for each patient, depending on comorbidities, disease status and response to vaccination. Individuals with CLL/lymphoma should continue social distancing and barrier measures. Systematic vaccination of their entourage and hospital workers would also directly benefit patients. If COVID-19 infection occurs after vaccination, convalescent plasma therapy ${ }^{14}$ or neutralizing monoclonal antibodies against SARS-CoV- $2^{15}$ could be discussed as future strategies for CLL/lymphoma patients with a low humoral immune response. Finally, prophylactic administration of neutralizing monoclonal antibodies is currently recommended in France for vaccinated seronegative patients and should also be evaluated.

\section{Authors}

Milena Kohn, ${ }^{1 *}$ Marc Delord, ${ }^{2 *}$ Maureen Chbat, ${ }^{1 *}$ Amina Guemriche, Fatiha Merabet, Anne-Laure Roupie, ${ }^{1}$ Naelle Lombion, ${ }^{3}$ Hassan Farhat, ${ }^{1}$ Thomas Longval, ${ }^{1}$ Aurélie Cabannes-Hamy, Juliette Lambert, ${ }^{1}$ Stéphanie Marque-Juillet, ${ }^{4}$ Victoria Raggueneau, ${ }^{4}$ Jennifer Osman, ${ }^{4}$ Marc Spentchian, ${ }^{4}$ Sophie Rigaudeau, ${ }^{1}$ Philippe Rousselot ${ }^{1}$ and Caroline Besson ${ }^{1.5}$

'Service d'Hématologie Oncologie, Centre Hospitalier de Versailles, Le Chesnay; ${ }^{2} \mathrm{DRCl}$, Centre Hospitalier de Versailles, Le Chesnay; ${ }^{3}$ Service d'hématologie, Hôpital de Poissy, Poissy; ${ }^{4}$ Service de Biologie, Centre Hospitalier de Versailles, Le Chesnay and ${ }^{5}$ UVSQ, Inserm, CESP, Villejuif, France

*MK, MD and MC contributed equally as co-first authors.

Correspondence:

Caroline Besson - cbesson@ch-versailles.fr

https://doi.org/10.3324/haematol.2021.280026

Received: September 17, 2021.

Accepted: November 4, 2021.

Prepublished: November 18, 2021.

\section{Disclosures}

No conflicts of interest to disclose. 


\section{Contributions}

MK, MC, AG, FM, ALR, NL, HF, TL ACH, JL, SR and PR included the patients, collected the data, and approved the article. MD performed the statistical analysis. $C B$ and $M D$ designed the study. MK, MD and CB wrote the article. SMJ, JO, VR and MS performed the biological analyses.

\section{Acknowledgments}

We thank the Centre Hospitalier de Versailles for their contribution to editing.

\section{References}

1. Duléry R, Lamure S, Delord M, et al. Prolonged in-hospital stay and higher mortality after Covid-19 among patients with nonHodgkin lymphoma treated with B-cell depleting immunotherapy. Am J Hematol. 2021;96(8):934-944.

2. Mato AR, Roeker LE, Lamanna N, et al. Outcomes of COVID-19 in patients with CLL: a multicenter international experience. Blood. 2020;136(10):1134-1143.

3. Herishanu Y, Avivi I, Aharon A, et al. Efficacy of the BNT162b2 mRNA COVID-19 vaccine in patients with chronic lymphocytic leukemia. Blood. 2021;137(23):3165-3173.

4. Benjamini O, Rokach L, Itchaki G, et al. Safety and efficacy of BNT162b mRNA COVID-19 vaccine in patients with chronic lymphocytic leukemia. Haematologica. 2022;107(3):625-634.

5. Gurion R, Rozovski U, Itchaki G, et al. Humoral serologic response to the BNT162b2 vaccine is abrogated in lymphoma patients within the first 12 months following treatment with anti-CD20 antibodies. Haematologica. 2022;107(3):715-720.

6. Perry C, Luttwak E, Balaban R, et al. Efficacy of the BNT162b2 mRNA COVID-19 vaccine in patients with B-cell non-Hodgkin lymphoma. Blood Adv. 2021;5(16):3053-3061.

7. Caillard S, Chavarot N, Bertrand D et al. Occurrence of severe COVID-19 in vaccinated transplant patients. Kidney Int. 2021;100(2):477-479.

8. Kamar N, Abravanel F, Marion O, Couat C, Izopet J, Del Bello A. Three doses of an mRNA Covid-19 vaccine in solid-organ tran- splant recipients. N Engl J Med. 2021;385(7):661-662.

9. Hall VG, Ferreira VH, Ku T, et al. Randomized trial of a third dose of mRNA-1273 vaccine in transplant recipients. N Engl J Med. 2021;385(13):1244-1246.

10. Prendecki M, Clarke C, Edwards $\mathrm{H}$, et al. Humoral and T-cell responses to SARS-CoV-2 vaccination in patients receiving immunosuppression. Ann Rheum Dis. 2021;80(10):1322-1329.

11. Benucci M, Damiani A, Infantino $M$, et al. Presence of specific $T$ cell response after SARS-CoV-2 vaccination in rheumatoid arthritis patients receiving rituximab. Immunol Res. 2021;69(4):309-311.

12. Mrak D, Tobudic S, Koblischke M, et al. SARS-CoV-2 vaccination in rituximab-treated patients: $B$ cells promote humoral immune responses in the presence of T-cell-mediated immunity. Ann Rheum Dis. 2021;80(10):1345-1350.

13. Marchesi F, Pimpinelli F, Giannarelli D, et al. Impact of anti$\mathrm{CD} 20$ monoclonal antibodies on serologic response to BNT162b2 vaccine in $\mathrm{B}$-cell non-Hodgkin's lymphomas. Leukemia. 2022;36(2):588-590.

14. Hueso T, Pouderoux C, Péré $H$, et al. Convalescent plasma therapy for B-cell-depleted patients with protracted COVID-19. Blood. 2020;136(20):2290-2295.

15. Weinreich DM, Sivapalasingam S, Norton T, et al. REGN-COV2, a neutralizing antibody cocktail, in outpatients with Covid-19. N Engl J Med. 2021;384(3):238-251. 